\title{
Editorial: Cell Communication in Vascular Biology
}

\author{
Mauricio P. Boric ${ }^{1}$, Walter N. Durán ${ }^{2}$ and Xavier F. Figueroa ${ }^{1 *}$ \\ ${ }^{1}$ Departamento de Fisiología, Facultad de Ciencias Biológicas, Pontificia Universidad Católica de Chile, Santiago, Chile, \\ ${ }^{2}$ Department of Pharmacology, Physiology and Neuroscience, New Jersey Medical School, Rutgers, The State University of \\ New Jersey, Newark, NJ, United States
}

Keywords: vascular function, blood flow distribution, cell communication, endothelial cell, vascular smooth muscle cell, vascular diseases, cerebral circulation

\section{Editorial on the Research Topic}

\section{Cell Communication in Vascular Biology}

This Research Topic comprises 5 review articles and 10 original contributions in the field of cell communication in Vascular Biology. Contributors used a variety of experimental approaches and models, or exercised keen analysis of published reports, to assess intercellular signaling between different pairs of cell types, such as the endothelium with vascular smooth muscle (VSM), platelets, leukocytes, tumor cells, and trophoblasts; but also, VSM and macrophages or among complex systems like intact vessels, the neurovascular unit and placenta. Communication includes direct cell-cell contact by adhesion molecules or gap junctions; classic receptor-mediated paracrine signaling and intercellular genetic modification by non-coding RNAs (NcRNA) or microvesicles transfer; all of this in the context of physiological regulation and different pathologies.

Control of blood flow supply by the vascular system is essential to keep the homeostasis of each cell of the organism, and then, blood flow distribution must be dynamically regulated to match the changing metabolic demand of surrounding tissue. Although the amount of blood flow depends on the total cross-sectional area of arteries (i.e., total number and caliber) irrigating a particular territory, blood vessels are not inert conduits aimed to passively transport blood into the tissues, but they actually are complex, multicellular structures that must work as a unit to rapidly adjust the distribution of blood flow according to the minute-to minute cellular requirements (Segal et al., 2000; Segal, 2005). The vessel wall is mainly constituted by smooth muscle cells and endothelial cells; thus, cell-to-cell communication is fundamental for the fine synchronization of function between these two cell types along the length of the vessels (Segal, 2015). It should be noted that synchronization and coordination are accomplished by an intricate system of complementary signaling pathways, which involves the interaction of direct cell-to-cell communication via gap junctions and the release of autocrine/paracrine signals (Figueroa and Duling, 2009; Moncada and Higgs, 2018). The relevance of this interaction is highlighted by the fine coordination of endothelial and smooth muscle cell function observed in the control of vasomotor tone through gap junctions located at the myoendothelial junctions (i.e., myoendothelial gap junctions) and endotheliumderived relaxing signals, such as nitric oxide (NO), prostaglandins and an unidentified signal known as endothelium-derived hyperpolarizing factor (EDHF) (Busse et al., 2002). The regulation of these signaling pathways is elegantly depicted in this Research Topic by Schmidt and de Wit.

Gap junctions are made up by the association of two hemichannels provided by each adjacent cell and, in turn, hemichannels are formed by connexin proteins (Sáez et al., 2003; Molica et al., 2018). However, individual hemichannels can be functional and work as a complementary signaling pathway to pannexins, which form channels with similar characteristics to hemichannels. Both connexin hemichannels and pannexin channels can contribute to the intercellular vascular 
signaling by the release of autocrine/paracrine signals, such as ATP, NO, and PG (Begandt et al., 2017), as noted in the article of Kameritsch and Pogoda.

Coordination of endothelial and smooth muscle cell function does not only rely on the intercellular signaling generated in a particular vessel segment (i.e., radial communication), but also on the longitudinal communication along the whole vessel length. In addition, small arteries and arterioles form a complex network in the microcirculation, where different vessel segments must work in concert to regulate blood flow distribution by precise, well-integrated changes in the luminal diameter (Segal, 2005; Figueroa and Duling, 2009), as demonstrated by the conduction of vasomotor responses along the longitudinal axis of microvessels (Møller et al.).

In addition to the complex signaling interaction between the cells of the vascular wall, regulation of blood flow distribution in peripheral tissues depends on both local signals from the surrounding tissues and the autonomic nervous system, basically the sympathetic nervous system (Thomas and Segal, 2004; Westcott and Segal, 2013; Gaete et al., 2014). However, paradoxically, cerebral blood flow is mainly controlled by local signaling across the neurovascular unit (neurons, astrocytes and cell types of the vascular wall) through a communication mechanism known as neurovascular coupling or vasculoneuronal coupling, depending on the direction of the signaling, from neurons to vessels or from vascular cells to neurons, respectively (Moore and Cao, 2008; Muñoz et al., 2015; Kim et al., 2016; Iadecola, 2017). Importantly, the article of Presa et al. illustrates that pathological conditions, such as hypertension, may lead to alterations in these communication mechanisms with a subsequent cognitive decline.

Not only the amount of blood flow through different territories must be regulated according to demand, but the vascular wall constitutes a regulated barrier for transport or exclusion of small metabolites, proteins and circulating blood cells. In this context, brain function also relies on the control of neuronal environment by endothelial cells in the blood brain barrier (BBB) (Obermeier et al., 2013) and the functional integrity of the barrier can be stressed in inflammatory conditions, as shown by Ittner et al. It should be noted that endothelial cells are not only important for the correct $\mathrm{BBB}$ function, but also for the blood-labyrinth-barrier (BLB) integrity of the stria vascularis in the cochlea (Shi, 2016). Interestingly, connexin-mediated communication plays a central role in the BBB signaling (Gaete et al., 2014) and now the study of Zhang $J$. et al. extends these data, demonstrating the relevance of Cx43 in the BLB functional integrity and, consequently, in the hearing function. In addition to the regulation of endothelial barrier integrity and exchange functions, cell adhesion and transmigration are important features that may be altered in pathological conditions (Durán et al., 2010; Filippi, 2019). The mechanisms involved in the communication of the vascular wall with blood circulating cells are complex; and in this article collection, endothelial interaction with and transmigration of leucocytes and tumor cells in the peripheral circulation, and the role of $\mathrm{NO}$ in these processes is clearly detailed by Aguilar et al..
Two complementary compartments can be recognized in the vascular system, the arterial and venous circulations. Both compartments, in turn, are divided into two functionally different vascular territories: the macrocirculation and microcirculation, which are structurally designed to perform different functions in response to distinct stimuli. In this context, it is noteworthy that fine control of gene expression is essential to maintain the functional integrity of the different segments of the vascular system and, consistent with this notion, the progress of diverse vascular-related pathologies is associated with variations in the transcriptional profile (Lamont and Childs, 2006; Man et al., 2016). Therefore, a thorough analysis of the changes in the gene expression pattern is required to understand the morphological and functional modifications observed in diverse pathological and clinical conditions, which highlights the contribution of the studies reported in this article collection regarding vessel remodeling induced by drastic hemodynamic changes, by Zhang X. et al. in an aortic constriction model and Jie et al. in an arteriovenous fistula model. Likewise, in agreement with the relevance of the fine control of gene expression in vascular homeostasis, regulatory NcRNA, such as long non-coding RNAs (lncRNA) and microRNAs (miRNA), have been recognized to be key players in the control of endothelial and smooth muscle cell function (Balamurali and Stoll, 2020; Ono et al., 2020). Accordingly, NcRNAs have been found to be involved in the pathogenesis of several cardiovascular diseases, but also, they have been proposed as therapeutic target molecules, in line with the results presented in the articles of Cheng et al. in spine cord injury repair and $\mathrm{Su}$ et al. in diabetic neointimal hyperplasia. Interestingly, miRNAs can be transmitted through microvesicles (Van Niel et al., 2018), which is addressed in this article collection by Zheng et al., showing that miRNA release via endothelial microvesicles can be involved in the pulmonary vascular leakage and lung injury observed in sepsis.

Cardiovascular diseases are the leading cause of morbidity and mortality worldwide and endothelial cells play a fundamental role in the control of cardiovascular homeostasis by the synthesis and release of a wide spectrum of signals that modulate angiogenesis, inflammation, hemostasis, vasomotor tone, and vascular permeability (Sturtzel, 2017). Then, in agreement with the importance of the endothelium, disturbance, impairment, or loss of normal function of endothelial cells (i.e., endothelial dysfunction) has been recognized as one of the most prominent risk factors for the development of cardiovascular diseases (Endemann and Schiffrin, 2004; Hirase and Node, 2012). Endothelial dysfunction has been directly linked with the initiation and evolution of hypertension and atherosclerosis (Gimbrone and García-Cardeña, 2016; Konukoglu and Uzun, 2016), but it has also been associated with physiological as well as pathophysiological processes, including aging, heart failure, coronary syndrome, thrombosis, intravascular coagulation, type I and II diabetes, obesity, inflammation, sepsis, and sleep apnea syndrome (Endemann and Schiffrin, 2004). In this article collection, the role of endothelial dysfunction in pre-eclampsia is highlighted and strengthened by the work of Liu et al.. However, it should be 
noted that vascular homeostasis and integrity also depend on the smooth muscle layer of the vessel wall (Majesky, 2016); in this context, Beck et al. address the impact of endothelial dysfunction in arterial remodeling in diabetes. Similarly, Ackers et al. show that alterations in smooth muscle cell signaling pathways can lead to the development of vascular diseases, such as atherosclerosis.

Overall, this Research Topic provides a comprehensive review and original contributions for the continued investigation of regulation of cell communications in vascular biology.

\section{REFERENCES}

Balamurali, D., and Stoll, M. (2020). Non-coding RNA databases in cardiovascular research. Non-coding RNA 6, 1-13. doi: 10.3390/ncrna6030035

Begandt, D., Good, M. E., Keller, A. S., DeLalio, L. J., Rowley, C., Isakson, B. E., et al. (2017). Pannexin channel and connexin hemichannel expression in vascular function and inflammation. BMC Cell Biol. 18, 1-16. doi: 10.1186/s12860-016-0119-3

Busse, R., Edwards, G., Félétou, M., Fleming, I., Vanhoutte, P. M., and Weston, A. H. (2002). EDHF: Bringing the concepts together. Trends Pharmacol. Sci. 23, 374-380. doi: 10.1016/S0165-6147(02)02050-3

Durán, W. N., Breslin, J. W., and Sánchez, F. A. (2010). The NO cascade, eNOS location, and microvascular permeability. Cardiovasc. Res. 87, 254-261. doi: $10.1093 / \mathrm{cvr} / \mathrm{cvq} 139$

Endemann, D. H., and Schiffrin, E. L. (2004). Endothelial dysfunction. J. Am. Soc. Nephrol. 15, 1983-1992. doi: 10.1097/01.ASN.0000132474.50966.DA

Figueroa, X. F., and Duling, B. R. (2009). Gap Junctions in the control of vascular function. Antioxid. Redox Signal. 11, 251-266. doi: 10.1089/ars.2008. 2117

Filippi, M. D. (2019). Neutrophil transendothelial migration: updates and new perspectives. Blood 133, 2149-2158. doi: 10.1182/blood-2018-12844605

Gaete, P. S., Lillo, M. A., and Figueroa, X. F. (2014). Functional role of connexins and pannexins in the interaction between vascular and nervous system. J. Cell. Physiol. 229, 1336-1345. doi: 10.1002/jcp.24563

Gimbrone, M. A., and García-Cardeña, G. (2016). Endothelial cell dysfunction and the pathobiology of atherosclerosis. Circ. Res. 118, 620-636. doi: 10.1161/CIRCRESAHA.115.306301

Hirase, T., and Node, K. (2012). Endothelial dysfunction as a cellular mechanism for vascular failure. Am. J. Physiol. Hear. Circ. Physiol. 302, H499-H505. doi: 10.1152/ajpheart.00325.2011

Iadecola, C. (2017). The neurovascular unit coming of age: a journey through neurovascular coupling in health and disease. Neuron 96, 17-42. doi: 10.1016/j.neuron.2017.07.030

Kim, K. J., Diaz, J. R., Iddings, J. A., and Filosa, J. A. (2016). Vasculo-neuronal coupling: retrograde vascular communication to brain neurons. J. Neurosci. 36, 12624-12639. doi: 10.1523/JNEUROSCI.1300-16.2016

Konukoglu, D., and Uzun, H. (2016). "Endothelial dysfunction and hypertension," in Hypertension: From Basic Research to Clinical Practice. Advances in Experimental Medicine and Biology, Vol. 956, ed M. S. Islam (Cham: Springer), 511-540. doi: 10.1007/5584_2016_90

Lamont, R. E., and Childs, S. (2006). MAPping out arteries and veins. Sci. STKE 2006:e39. doi: 10.1126/stke.3552006pe39

Majesky, M. W. (2016). Vascular smooth muscle cells. Arterioscler. Thromb. Vasc. Biol. 36, e82-e86. doi: 10.1161/ATVBAHA.116.308261

Man, H. S. J., Yan, M. S., Lee, J. J. Y., and Marsden, P. A. (2016). Epigenetic determinants of cardiovascular gene expression: vascular endothelium. Epigenomics 8, 959-979. doi: 10.2217/epi-2016-0012

Molica, F., Figueroa, X. F., Kwak, B. R., Isakson, B. E., and Gibbins, J. M. (2018). Connexins and pannexins in vascular function and disease. Int. J. Mol. Sci. 19:1663. doi: 10.3390/ijms19061663

\section{AUTHOR CONTRIBUTIONS}

XF: manuscript writing and edition. $\mathrm{MB}$ and WD: contribution in manuscript writing and edition. All authors contributed to the article and approved the submitted version.

\section{FUNDING}

This work was supported by Grant \#1150530 (to XF) from Fondo Nacional de Desarrollo Científico y Tecnológico (FONDECYT) and by NIH-NHLBI grant 1R01HL146539 (to WD).

Moncada, S., and Higgs, E. A. (2018). Molecular mechanisms and therapeutic strategies related to nitric oxide. FASEB J. 9, 1319-1330. doi: 10.1096/fasebj.9.13.7557022

Moore, C. I., and Cao, R. (2008). The hemo-neural hypothesis: on the role of blood flow in information processing. J. Neurophysiol. 99, 2035-2047. doi: 10.1152/jn.01366.2006

Muñoz, M. F., Puebla, M., and Figueroa, X. F. (2015). Control of the neurovascular coupling by nitric oxide-dependent regulation of astrocytic $\mathrm{Ca}^{2+}$ signaling. Front. Cell. Neurosci. 9:59. doi: 10.3389/fncel.2015.00059

Obermeier, B., Daneman, R., and Ransohoff, R. M. (2013). Development, maintenance and disruption of the blood-brain barrier. Nat. Med. 19, 1584-1596. doi: 10.1038/nm.3407

Ono, K., Horie, T., Baba, O., Kimura, M., Tsuji, S., Rodriguez, R. R., et al. (2020). Functional non-coding RNAs in vascular diseases. FEBS J. doi: 10.1111/febs.15678. [Epub ahead of print].

Sáez, J. C., Berthoud, V. M., Brañes, M. C., Martínez, A. D., and Beyer, E. C. (2003). Plasma membrane channels formed by connexins: their regulation and functions. Physiol. Rev. 83, 1359-1400. doi: 10.1152/physrev.00007.2003

Segal, S. S. (2005). Regulation of blood flow in the microcirculation. Microcirculation 12, 33-45. doi: 10.1080/10739680590895028

Segal, S. S. (2015). Integration and modulation of intercellular signaling underlying blood flow control. J. Vasc. Res. 52, 136-157. doi: 10.1159/000439112

Segal, S. S., John, T., and Haven, N. (2000). Integration of blood flow control to skeletal muscle: key role of feed arteries. Acta Physiol. Scand. 168, 511-518. doi: 10.1046/j.1365-201x.2000.00703.x

Shi, X. (2016). Pathophysiology of the cochlear intrastrial fluid-blood barrier (review). Hear. Res. 338, 52-63. doi: 10.1016/j.heares.2016.01.010

Sturtzel, C. (2017). "Endothelial cells," in The Immunology of Cardiovascular Homeostasis and Pathology. Advances in Experimental Medicine and Biology, Vol 1003, eds S. Sattler and T. Kennedy-Lydon (Cham: Springer), 71-91. doi: 10.1007/978-3-319-57613-8_4

Thomas, G. D., and Segal, S. S. (2004). Neural control of muscle blood flow during exercise. J. Appl. Physiol. 97, 731-738. doi: 10.1152/japplphysiol.00076.2004

Van Niel, G., D'Angelo, G., and Raposo, G. (2018). Shedding light on the cell biology of extracellular vesicles. Nat. Rev. Mol. Cell Biol. 19, 213-228. doi: 10.1038/nrm.2017.125

Westcott, E. B., and Segal, S. S. (2013). Perivascular innervation: a multiplicity of roles in vasomotor control and myoendothelial signaling. Microcirculation 20, 217-238. doi: 10.1111/micc.12035

Conflict of Interest: The authors declare that the research was conducted in the absence of any commercial or financial relationships that could be construed as a potential conflict of interest.

Copyright (c) 2021 Boric, Durán and Figueroa. This is an open-access article distributed under the terms of the Creative Commons Attribution License (CC BY). The use, distribution or reproduction in other forums is permitted, provided the original author(s) and the copyright owner(s) are credited and that the original publication in this journal is cited, in accordance with accepted academic practice. No use, distribution or reproduction is permitted which does not comply with these terms. 